\title{
Article
}

\section{Attention "Blinks" Differently for Plants and Animals}

\section{Benjamin Balas* and Jennifer L. Momsen ${ }^{\dagger}$}

\author{
${ }^{*}$ Psychology Department, Center for Visual and Cognitive Neuroscience, and ${ }^{\dagger}$ Department of Biological \\ Sciences, North Dakota State University, Fargo, ND 58108
}

Submitted May 2, 2014; Revised June 19, 2014; Accepted June 19, 2014

Monitoring Editor: Emily A. Holt

\begin{abstract}
Plants, to many, are simply not as interesting as animals. Students typically prefer to study animals rather than plants and recall plants more poorly, and plants are underrepresented in the classroom. The observed paucity of interest for plants has been described as plant blindness, a term that is meant to encapsulate both the tendency to neglect plants in the environment and the lack of appreciation for plants' functional roles. While the term plant blindness suggests a perceptual or attentional component to plant neglect, few studies have examined whether there are real differences in how plants and animals are perceived. Here, we use an established paradigm in visual cognition, the "attentional blink," to compare the extent to which images of plants and animals capture attentional resources. We find that participants are better able to detect animals than plants in rapid image sequences and that visual attention has a different refractory period when a plant has been detected. These results suggest there are fundamental differences in how the visual system processes plants that may contribute to plant blindness. We discuss how perceptual and physiological constraints on visual processing may suggest useful strategies for characterizing and overcoming zoocentrism.
\end{abstract}

\section{INTRODUCTION}

Botanists have long observed and lamented the routine neglect of plants in textbooks (Link-Pérez et al., 2009; Uno, 2009; Schussler et al., 2010), teaching laboratories (Uno, 2009), and lectures (Darley, 1990; Bozniak, 1994; Uno, 1994). Students from kindergarten through college can better recall and name animals (Bebbington, 2005; Schussler and Olzak, 2008; Patrick and Tunnicliffe, 2011) and prefer to study animals (Wandersee, 1986; Marbach-Ad, 2004). Perhaps not surprisingly, undergraduate botany majors are declining nationally (Uno, 1994; Hershey, 1996) to the extent that data on botany majors are no longer reported by the National Center for Education Statistics.

DOI: $10.1187 /$ cbe. $14-05-0080$

Address corresponding to: Jennifer L. Momsen (jennifer.momsen@ ndsu.edu).

(C) 2014 B. Balas and J. L. Momsen. CBE-Life Sciences Education (C) 2014 The American Society for Cell Biology. This article is distributed by The American Society for Cell Biology under license from the author(s). It is available to the public under an AttributionNoncommercial-Share Alike 3.0 Unported Creative Commons License (http:/ / creativecommons.org/licenses/by-nc-sa/3.0).

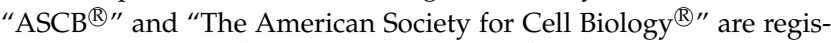
tered trademarks of The American Society for Cell Biology.
Why are plants typically ignored and neglected by students, teachers, and the general public? Initial hypotheses focused on education, labeling the undergraduate life sciences curriculum as zoocentric or zoochauvinistic-that is, instruction and textbooks reflect a pervasive belief that studying animals is more important than studying plants (e.g., Darley, 1990; Flannery, 1991; Bozniak, 1994; Hershey, 1996, 2002). Indeed, elementary school science textbooks include more animal content, as evidenced in the number and diversity of images, the quantity of text, and the number of animal examples of core biological concepts (Link-Pérez et al., 2009; Schussler et al., 2010). However, such claims of zoocentrism imply that instructors and textbook authors actively choose to omit plant examples in favor of animals. While certainly plausible, there is no evidence to support such claims; indeed, it is possible that instructors and authors are implicitly biased against plants; that is, they are unaware that they include substantially fewer plant examples in their curricula.

The pervasiveness of plant neglect in not only the undergraduate and high school biology curriculum but in society in general prompted several researchers to consider a broader explanatory mechanism. Wandersee and Schussler (1999, 2001), drawing on visual cognition research, introduced the idea of plant blindness. As defined by Wandersee and Schussler (1999), plant blindness is both the inability to 
notice plants in their environment and a failure to recognize and appreciate the utility of plants to life on earth coupled with a belief that plants are somehow inferior to animals. This multifaceted definition recognizes cultural contributions to plant blindness, while also introducing the idea that visual perception may limit people's ability to see and, by extension, value plants.

Experimental support of plant blindness as a perceptual or cognitive phenomenon is currently limited to a single study. Schussler and Olzak (2008) presented undergraduate psychology and botany students with a series of plant and animal images to test the hypothesis that undergraduate students would recall more animal than plant images. After viewing the images, students were briefly distracted before being asked to list as many of the plant and animal images they could recall. Students, even botany students, recalled more animals than plants. These results suggest that plants are to some extent more weakly encoded than animals.

Such results are promising, but limited. Because the K-16 life sciences curriculum appears to overrepresent animals, as evidenced by textbook studies (Uno, 1994; Link-Pérez et al., 2009; Schussler et al., 2010), it is possible that students have built a robust mental infrastructure that better supports the recall of animals over plants, even when images of very common plants like pumpkin, corn, and carrot are used. Thus, a recall task measures the effect of an already zoocentric curriculum on students' ability to remember organisms.

Recalling or naming a plant image is inherently an act of remembering, not perceiving. That is, whether or not a particular item in a previously studied set of stimuli is recalled later depends on a range of factors, including the visual similarity of the target item to distractors, the phonological similarity of the target item's name to distractors, the visual salience of the target item, and so on. Even if we assume that these properties are well matched for plant images and animal images in a memory task, it is difficult to determine from this result what aspects of visual memory may differ for plants and animals. During retention (the interval when information about an item to be remembered must be maintained-usually a delay period between study and test), do plant images decay more rapidly than animals? Another possibility is that plant images are not robustly encoded in the first place: the visual description of a plant that is to be remembered may not be as complete or rich as that of an animal that is to be remembered. Finally, does plant blindness reflect a failure to adequately store information about plants, or is there some failure to adequately see or attend to plants in the first place? By attention, we refer to observers' ability to select some subset of the environment for further processing (e.g., singling out a particular object to study more closely; for technical definitions of these terms and others related to visual memory, see Baddeley et al., 2009). We suggest that experimental studies that investigate this latter question, whether or not observers have differential abilities to perceive plants and animals, are desperately needed.

In response to this need, we have adapted techniques from research in visual perception to provide empirical evidence that our attention is captured differently by plants than by animals. Specifically, we demonstrate that visual attention to plants is deployed differently than to animals, leading to measurable differences in how well attention can be redeployed following plant versus animal detection. To show this, we have taken advantage of an established phenomenon in visual perception known as the "attentional blink," which is a particularly useful meas of objectively measuring the extent to which people detect plants and animals in a quantitatively different way.

\section{ATTENTIONAL BLINK}

"Attentional blink" (or $\mathrm{AB}$ ) is a robust phenomenon in visual perception in which detecting the first of two targets in a sequence of rapidly presented images compromises the ability to detect the second target for a short time (Raymond et al., 1992; for a review, see Shapiro et al., 1997). Typically, participants in $\mathrm{AB}$ tasks are asked to perform a dual-target detection task: within a sequence of letters presented very rapidly (10-50 ms per letter), participants might be asked to report the presence of either the letter " $\mathrm{T}$ " or the letter " $\mathrm{X}$," for example. On any given trial, an instance of the first target (referred to as T1) or the second target (T2) may or may not be present. In general, participants are successful at reporting the presence or absence of T1 accurately; that is, visual detection and recognition, even for complex targets (Potter, 1976; Potter et al., 2013), is highly efficient. However, the successful detection of T2 depends critically on T1. If T1 is absent, T2 is typically reported accurately. If T1 is present, however, and if T2 appears in the image sequence within $\sim 500 \mathrm{~ms}$ of T1's appearance, $\mathrm{T} 2$ is less frequently reported as being present. This time-dependent decrement in T2 detectability is typically accounted for by describing visual attention as a limited resource. The first target captures visual attention. Disengaging from the target takes time (thus freeing up resources for visual attention), meaning that subsequent targets appearing too close to the first are frequently missed, because there are insufficient resources available. In other words, attention "blinks."

The nature of T1's impact on T2 has been used to define properties of visual attention in general (Vul et al., 2008) and also to quantify the extent to which different target categories drive visual attention exogenously. That is, even though attention blinks, are some targets so salient that they are better detected than others even if they appear during the post-T1 refractory period? In some reports, T2 targets that are highly salient, because they are visually dissimilar to T1 (Raymond et al., 1995) or because they depict arousing words or images, can overcome the AB (e.g., emotionally charged words; Keil and Ihssen, 2004), suggesting that stimulus properties can sometimes overcome some of the intrinsic limitations on visual attention.

In the current study, we adopt similar logic to determine whether plants capture attention less effectively than animals, using $\mathrm{AB}$ as a means of quantifying attentional engagement. Specifically, we designed an AB task in which plants or animals were used as T1 targets, so we could measure the relative impact of target images in each category on the subsequent detection of unrelated T2 targets (pictures of water). Rather than examine what factors may contribute to distinct $\mathrm{T} 2$ targets being more detectable than others during the $A B$, our hypothesis was that differences in attentional engagement for plants versus animals (possibly as a result of "plant blindness") should lead to differences in the impact of plant/animal T1 targets on T2 detection. That is, if 
plant blindness is the result of a failure of visual attention, reduced attention to plants should be manifest in our $A B$ task as improved detection of T2 targets when plants are used as T1 targets relative to when animals are used. The standard $\mathrm{AB}$ paradigm also affords us the opportunity to examine potential differences in T1 detection for plants and animals, an additional measure of how detectable plants and animals were in rapid sequences of images. Our study is a novel application of theories and paradigms from visual cognition to examine the possible perceptual basis of plant blindness.

\section{METHODS}

\section{Subjects}

We recruited 24 participants (14 female) from the North Dakota State University Introductory Psychology study pool, which allows students to sign up online for a variety of ongoing experiments being conducted by researchers in the psychology department. Students sign up by reviewing the available studies and choosing studies in which they wish to participate. Introductory Psychology satisfies general education requirements and is thus composed of students from a wide variety of majors. Participants received course credit (3 credits toward a 15-credit component of the class) or were paid ( $\$ 10 / \mathrm{h}$ prorated) for taking part in the study and gave informed consent before beginning the study. All participants were ages 18-24 years old, had normal or corrected-to-normal vision, and had no known visual or neurological impairments. After consent was obtained, participants were told that the goal of this study was to determine observers' ability to detect different kinds of images in sequences of rapidly presented images and the dual-target detection paradigm was explained to them.

\section{Stimuli}

Our stimulus set was made up of full-color images of natural objects and materials belonging to a number of distinct categories. Distractor images (stimuli that were never detection targets) were composed of images of stone and plastic drawn from the Flickr Materials Database (Sharan et al., 2009). Target images of plant foliage were taken from the same database; we excluded any images that included an animal (e.g., a bee hovering near a flower), were focused exclusively on a single flower, or were of dead plant matter (e.g., logs, dried leaves). Because the Flickr Materials Database did not have a suite of animal images, target images of animals were collected from a variety of online sources and depicted a range of species. Finally, target images of water were also drawn from the Flickr Materials Database. Our full stimulus set was made up of 50 images per category, and all images were cropped or resized to be $512 \times 384$ pixels in size. T1 and T2 targets were selected such that no image contained an instance of multiple categories that were task-relevant for an individual participant: images of plant and animals did not contain water, for example. This is critically important, because we did not want participants to be confused about whether or not an image containing a plant and water should be considered as a "plant" detection, a "water" detection, or both. We note that some images of animals did include plants, but because plant and ani- mal targets were presented to different groups of participants, these images did not introduce any task ambiguity for our observers and thus should not have impacted performance. The full set of stimuli used in this task can be obtained by visiting the following URL: https: / /dl.dropboxusercontent.com/ u/4961099/BalasMomsen_AB_Stimuli.zip.

\section{Procedure}

We adopted a standard AB paradigm (Shapiro et al., 1997) to examine the extent to which detecting plants and animals in a rapidly presented sequence of images affected the subsequent detection of a second target in the sequence. We presented participants with a series of image sequences, each composed of 24 images. The participants' task was to view each sequence and determine whether or not the sequence contained an image belonging to either of two predetermined target categories. Half of our participants were asked to detect images of plants and images of water ("plant" condition), while the remaining half were asked to detect images of animals and water ("animal" condition). Throughout the text, we shall refer to images of plants and animals as "T1" targets and images of water as "T2" targets.

On each trial, image sequences were constructed such that T1 targets (if present) would appear within the middle third of the sequence (frames 9-16 of the 24-frame sequence), with the specific position determined on each trial via independent uniform sampling of the relevant sequence interval. T2 targets (if present) appeared with equal frequency at one of eight "lag positions" following the T1 target. Specifically, T2 targets could appear either 1-8 frames after a T1 target. For image sequences in which a T2 target was present, but no T1 target appeared, T2 position was matched across trials in which T1 and T2 were both present. Our full design was thus defined by all combinations of T1 presence/absence, T2 presence/absence, and the eight lag positions that determined T2 position relative to T1 (Figure 1). The specific images comprising each sequence were randomly sampled from the relevant categories before each trial. Participants completed 20 repetitions of each cell in the design, yielding a total of 640 trials per experimental session. The full experimental session was split into two blocks of 320 trials, so participants had the opportunity to take a short break. Trials were presented in a fully randomized order for each participant.

Participants completed this task on a MacBook laptop with a display refresh rate of $60 \mathrm{~Hz}$. Participants sat in a darkened room with the display positioned at a comfortable height. Viewing distance was $\sim 40 \mathrm{~cm}$ and our stimuli subtended $\sim 6$ degrees of visual angle at this distance. Within an image sequence, each individual image was displayed for $\sim 32 \mathrm{~ms}$, with an interstimulus interval of $\sim 70 \mathrm{~ms}$ (in other words, the first image following T1 appears $102 \mathrm{~ms}$ following T1 onset; subsequent images appear $102 \mathrm{~ms}$ after onset of the previous image). Following the completion of each sequence, participants were presented with two response screens: The first screen asked participants: "Did you see any [plants/animals]?"; the second screen asked: "Did you see any water?" Participants had unlimited time to respond to these questions and were asked to respond to each screen by using the " $\mathrm{y}$ " and " $\mathrm{n}$ " keys to indicate "yes" or "no," respectively. 
A.

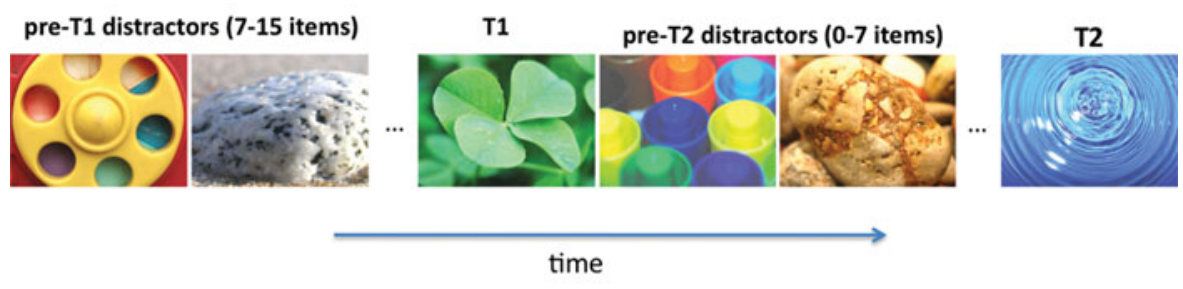

\section{B.}

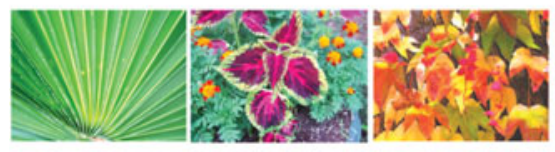

Plant T1 images

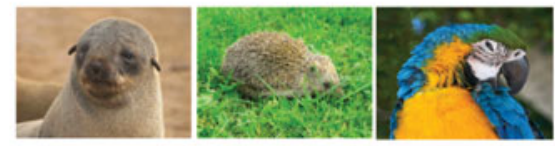

Animal T1 images
Figure 1. (A) Schematic view of a single trial in our $\mathrm{AB}$ task. Participants were asked to perform a dual-target task in which they were to report whether or not they saw an image of a plant/animal (target T1) and whether or not they saw an image of water (target T2). Both T1 and T2 could be present or absent on any given trial, but T1 would always precede T2 if both were present. In typical AB studies, the ability to detect $\mathrm{T} 2$ is affected by its presence in the sequence relative to $\mathrm{T} 1$, so we varied their relative positions across trials. (B) Examples of plant and animal images we used as T1 targets in separate participant groups.

\section{RESULTS}

Overall, we were interested in two different ways of comparing the extent to which images of plants and animals captured participants' attention in a rapidly presented image sequence. First, how well do participants detect plants versus animals? Second, how does the presence of a plant or animal impact the detection of a subsequently presented target that belongs to neither category?

\section{T1 Detection: Hit Rates and False-Alarm Rates}

To address the first question, we compared T1 detectability between our "plant" and "animal" groups using both hit rates (successfully detecting T1 when it was present) and falsealarm rates (indicating T1 was present when it was not). We calculated the $\mathrm{T} 1$ hit rate and false-alarm rate for each participant and compared the values obtained from the plant group and the animal group via a two-tailed independentsamples $t$ test (Figure 2). We found that hit rates differed sig-

\section{T1 Detection: Hit and False Alarm Rates}

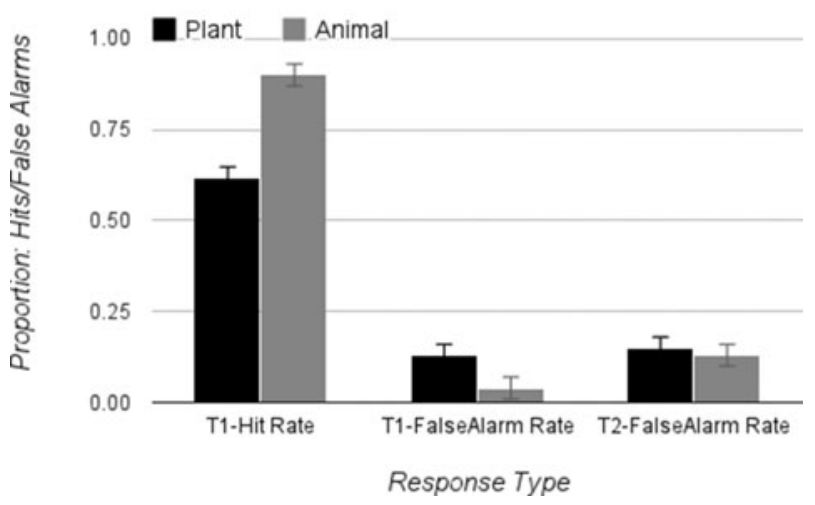

Figure 2. Participants in the plant group were in general less sensitive to $\mathrm{T} 1$ targets than participants in the animal group. Hit rates were significantly lower for plant targets than animal targets, and false-alarm rates were higher for plants than for animals. We have also included the average T2 false-alarm rate, which did not differ across participant groups. nificantly $(t(22)=-3.94, p<0.001)$ between the two groups, such that hit rates were lower in the plant group $($ mean $=0.69$, $\mathrm{SEM}=0.048$ ) than in the animal group (mean $=0.90, \mathrm{SEM}=$ 0.020 ). We also found that false-alarm rates significantly differed between the two groups $(t(22)=2.33, p=0.029)$, such that participants in the plant group made more false alarms (mean $=0.12, \mathrm{SEM}=0.032)$ than participants in the animal group $($ mean $=0.04, \mathrm{SEM}=0.013)$. Both outcomes suggest that plant images are harder to detect than animal images: participants both see plants less often when they are present and more frequently report that plants occur when they do not.

To confirm that the observed differences in T1 detection were not simply the result of plant images that were ambiguous, we ran an additional norming study to determine how categorizable our plant images were as plants. We recruited 24 participants via the Amazon Mechanical Turk (a crowdsourcing tool that is widely used to obtain ratings for psychological stimuli, among other applications) to view our plant images and indicate whether or not each image depicted a plant or not. Participants were free to view each image for as much time as they liked and labeled each image as plant or "not a plant." We found that only one image from our stimulus set was labeled as a plant by fewer than 23 of our 24 observers-this single image was labeled as "not a plant" by four observers. Overall, these results strongly suggest that all but one of our plant images were easily categorizable as plants, and the impact of this single image out of a set of 50 is unlikely to be substantial. We therefore conclude that the differences we observed between plant and animal T1 detection rates are not simply the result of ambiguous plant images.

\section{T2 Hit Rates}

To address our second question, we compared T2 hit rates across the plant and animal groups as a function of T1 presence/absence and the lag between T2 and T1 when T1 was present. In general, the presence of $\mathrm{T} 1$ is expected to negatively impact hit rates within some temporal window following T1 onset: T2 should be easier to detect when T1 did not appear earlier in the sequence. Our question is whether or not the impact on $\mathrm{T} 2$ hit rates differs according to whether 
T2 Hit Rates vs. Lag

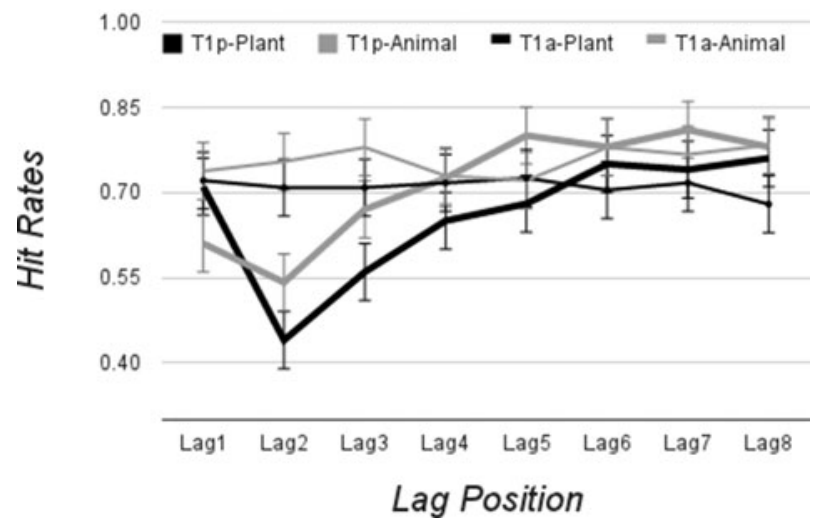

Figure 3. Average T2 hit rate for plant and animal participants as a function of whether T1 was present or absent and T2 lag position. When T1 was absent (T1a, thin lines), T2 hit rates did not vary significantly across lag position. When T1 was present (T1p, thick lines), T2 hit rates dipped and then recovered as lag position increased. Black lines depict data from plant trials; gray lines depict data from animal trials.

or not $\mathrm{T} 1$ is a plant or an animal. We calculated the T2 hit rate for each participant at each lag position for T1-present and T1-absent trials (Figure 3). Critically, we included only data from T1-present trials in which participants correctly indicated that T1 was present. Given the difference in T1 detection rates described above, this criterion helps to make it more likely that any category differences we observed in terms of T2 detection are the result of differences in the AB itself, rather than an artifact of poorer detection of T1. This also helps ameliorate some of the concerns regarding image-level differences between our plant and animal images, since we analyze only trials in which $\mathrm{T} 1$ was sufficiently salient and categorizable to be correctly detected. We submitted these values to a $2 \times 2 \times 8$ mixed-design analysis of variance with T1 presence and lag position as within-subject factors and participant group (plant vs. animal) as a between-subjects factor.

Our analysis revealed a main effect of lag position $(F(7,16)$ $\left.=4.046, p=0.01, \eta^{2}=0.64\right)$, but no main effect of participant group $(F(1,22)=0.68, p=0.42)$. The main effect of lag position was the result of significantly lower $\mathrm{T} 2$ hit rates at a lag position of 2 (mean $=0.61, \mathrm{SEM}=0.04)$ compared with lag positions $5-8$ (mean $\sim 0.76, \mathrm{SEM} \sim 0.03$ in each case), which simply confirms the presence of an $\mathrm{AB}$.

The main effect of lag was qualified by two interactions. First, we observed a significant interaction between T1 presence and lag position $\left(F(7,16)=7.38, p<0.001, \eta^{2}=0.76\right)$, such that T2 hit rates were essentially flat across lag position when T1 was absent, but varied when T1 was present. Critically, however, this was further qualified by a significant threeway interaction between participant group, T1 presence, and lag position (quadratic model $\left[F(1,22)=7.86, p=0.010, \eta^{2}=\right.$ $0.26]$ ), indicating that the interaction of $\mathrm{T} 1$ presence and lag position differs between plant and animal groups. The use of a quadratic contrast for this effect is appropriate given the known U-shaped function that is typical of the AB (Shapiro et al., 1997; Quinn and Keough, 2002).

\section{DISCUSSION}

Participants' performance in our AB task suggests that our attention is not captured by plants in the same way as it is by animals. The difference in sensitivity we observed for plant versus animal $\mathrm{T} 1$ targets demonstrates that plant images are less reliably detected in an image sequence than animals (Figure 2); participants are more likely to miss plant images that are present and to falsely report seeing a plant when one was not present. Though adult observers are generally credited with impressive accuracy in rapid serial visual presentation tasks (Potter et al., 2013), our data suggest that some targets are more easily detected than others. Thus, plant blindness may result from differences in how attention is deployed to plants. Specifically, it is harder to notice plants.

Besides this difference in sensitivity to plants versus animals, we also observed differences in the AB for T2 targets that followed plant versus animal T1 targets (Figure 3). Broadly, our result is consistent with prior results demonstrating that $\mathrm{T} 2$ detection is modulated in some circumstances by how attention is allocated to other arousing or engaging items (Arnell et al., 2007). The differential impact of plant targets compared with animal targets suggests that visual attention has a different refractory period when a plant has been detected, which implies that attentional resources are deployed differently for plant targets. Animal targets appear to induce an immediate decrement in T2 detection, while plant targets do not appear to have an influence until later. The magnitude of the performance decrement for $\mathrm{T} 2$ detection appears to be more or less the same at its peak for plants and animals, but the peak does not arrive as quickly for plants. Put another way, attention to plants is delayed, but not reduced, relative to attention to animals (Vul et al., 2008). Considered together, the data from $\mathrm{T} 1$ and $\mathrm{T} 2$ target detection demonstrate that plants are not detected as robustly as animals in the first place, nor do they capture attention resources as quickly. Plant blindness thus may result in part from differences in the visual processing of plants versus animals.

An important caveat to these results is that, in this experiment, we did not attempt to closely match our plant and animal images in terms of low-level visual properties. That is, our images of plants potentially differ from our images of animals in terms of spatial frequency content, color and luminance histograms, and visual texture. This means that our results may be driven by some aspect of high-level visual processing (a difference in how plants and animals are processed at levels of the visual system that are largely robust to image-level appearance), or they may be driven by low-level properties of the images that covary with plant/animal categories (a difference in how plants vs. animals typically look that is not necessarily about plants per se). However, while this is important to acknowledge, so our results can be contextualized relative to other studies in the visual cognition literature, we suggest that the distinction between low-level and high-level contributions to our effect is not critically important for our purposes. In natural environments, observers routinely encounter real plants and animals that differ in lowlevel image properties. Our use of natural photographs that were also not closely matched thus reflects the perceptual and attentional processes that are engaged when observers see plants and animals out in the world. Thus, while it would be interesting to identify the perceptual dimensions (e.g., 
specific colors, the presence of eyes in animal faces) that may drive category differences in our AB task, the current results demonstrate there are intriguing differences in how natural images of plants and animals are perceived.

\section{IMPLICATIONS FOR INSTRUCTION}

Our results demonstrate a very real difference in human perception of plants and animals and support the inclusion of a visual perceptual component in the definition of plant blindness (Wandersee and Schussler, 1999, 2001). We are cautious, however, in extending these results beyond the definition of plant blindness. For example, several critics of plant blindness have stated the concept itself implies that zoocentric attitudes are both normal and inevitable for humans (Hershey, 2002; Hall, 2011). Whether plant blindness is normal and inevitable remains an open question; indeed, we might ask whether humans are blind to plants or simply more attentive to animals.

We would argue, however, that such disagreements draw attention from what botanists and educators should be focusing on, namely, the design of instruction that addresses plant blindness and helps students compensate for and overcome their perceptual limitations. For example, suggestions to strive for plant equality (Hershey, 2002; Schussler and Olzak, 2008), that is, increasing the representation of plants in textbook images and classroom examples, may be insufficient to counter a physiological basis for plant blindness. Uno (2009) previously addressed the need to build botanical instruction based on the learning sciences and disciplinebased education as a mechanism to target and combat plant blindness. Below, we offer several additional suggestions that emerge from our research on plant blindness.

First, because plant blindness is, in part, a physiological phenomenon, we suggest that instruction should directly define and address plant blindness. Beginning in introductory biology, students should learn what plant blindness is, including both the possible physiological underpinnings and cultural implications. As part of that instruction, students should learn that plants capture attention differently than animals and, as a result, they are unlikely to "see" plants in their daily lives. In response, students can then work to identify strategies to overcome or compensate for plant blindness.

Second, as noted previously, instruction about plants should reflect what we know about best practices in science, technology, engineering, and mathematics education (Uno, 2009), namely that constructivist learning environments (i.e., active learning) increase student learning (Freeman et al., 2014). Such classrooms go well beyond simply exposing students to additional plant examples. For example, targeted, place-based biodiversity instruction that engaged children in searching for, identifying, and investigating local flora increased both their recognition of and appreciation for plants (Lindemann-Matthies, 2002, 2005). These students actively attended to wild plants; we might, therefore, predict that these students are more likely to see plants in their daily lives.

Such instruction could easily translate into the undergraduate life sciences classroom. For example, observational lab experiences that make use of campus flora (e.g., Long and Wyse, 2012) would promote student engagement with and observation of local plants and may change how students attend to plants. In the lecture hall, plant blindness might also be combated by curricular reforms that focus on core biolog- ical concepts (American Association for the Advancement of Science, 2011). This restructuring provides a platform to integrate plants into the life sciences curriculum rather than teaching plants as "other" or completely different (Hall, 2011). Instruction on a particular biological principle, for example, energy acquisition, would use a suite of plant and animal examples to demonstrate the diversity in mechanisms used by organisms to acquire energy. In these classrooms, students would not only encounter more plant examples but would also learn to attend to plants in the same manner they attend to animals-as components of a living system.

Finally, we would suggest that botanical instruction should capitalize on the dual-coding theory of multimedia learning (Pavio, 1986; Mayer and Sims, 1994; Mayer, 2009). This theory assumes learners possess two main "channels" to process information (auditory and visual), and these channels are of limited capacity. Learning happens as a result of the learner actively selecting, organizing, and building mental representations from incoming information that is then integrated with prior knowledge (Mayer, 2009). Instruction that engages both auditory and visual channels, for example, presenting plant images simultaneously with text or narration, provides more opportunities for the learner to engage with botanical instruction, allows the learner to build robust mental models, and supports the integration of those models with prior knowledge, thus leading to meaningful learning about plants. Such deep learning about plants may act to foster student appreciation for plants while facilitating their ability to see and attend to plants in their daily lives.

Plant blindness is inherently a complex phenomenon. The cultural underpinnings of plant blindness have been well recognized; here, we add to a small body of research documenting a physiological mechanism underlying plant blindness. Our work supports the complete definition of plant blindness put forth by Wandersee and Schussler (1999) as encompassing both limits to human visual perception and cultural constraints. Further studies that clarify just how the human brain perceives and attends to plants can further inform instructional efforts and help learners take concrete steps toward overcoming plant blindness.

\section{ACKNOWLEDGMENTS}

This research was conducted in compliance with institutional review board regulations (IRB protocol SM11167). B.B. was supported by National Institute of General Medical Sciences grant P20 GM103505. Special thanks to Alyson Saville for her help recruiting and testing participants.

\section{REFERENCES}

American Association for the Advancement of Science (2011). Vision and Change in Undergraduate Biology Education: A Call to Action, Washington, DC.

Arnell KM, Killman KV, Fijavz D (2007). Blinded by emotion: target misses follow attention capture by arousing distractors in RSVP. Emotion 7, 465-477.

Baddeley A, Eysenck MW, Anderson MC (2009). Memory, Hove, UK: Psychology Press.

Bebbington A (2005). The ability of A-level students to name plants. J Biol Educ 39, 63-67. 
Bozniak EC (1994). Challenges facing plant biology teaching programs. Plant Sci Bull 40, 42-46.

Darley WM (1990). The essence of "plantness." Am Biol Teach 52, 354-357.

Flannery MC (1991). Considering plants. Am Biol Teach 53, 306-309.

Freeman S, Eddy SL, McDonough M, Smith MK, Okoroafor N, Jordt $\mathrm{H}$, Wenderoth MP (2014). Active learning increases student performance in science, engineering, and mathematics. Proc Natl Acad Sci USA $11,8410-8415$.

Hall M (2011). Plants as Persons: A Philosophical Botany, Albany, NY: SUNY Press.

Hershey DR (1996). A historical perspective on problems in botany teaching. Am Biol Teach 58, 340-347.

Hershey DR (2002). Plants rarely thermoregulate. Am Biol Teach 64 , 413.

Keil A, Ihssen N (2004). Identification facilitation for emotionally arousing verbs during the attentional blink. Emotion 4, 23-35.

Lindemann-Matthies P (2002). The influence of an educational program on children's perception of biodiversity. J Environ Educ 33, 22-31.

Lindemann-Matthies P (2005). "Loveable" mammals and "lifeless" plants: how children's interest in common local organisms can be enhanced through observation of nature. Int J Sci Educ 27, 655-677.

Link-Pérez MA, Dollo VH, Weber KM, Schussler EE (2009). What's in a name: differential labeling of plant and animal photographs in two nationally syndicated elementary science textbook series. Int J Sci Educ 32, 1227-1242.

Long TM, Wyse SA (2012). A season for inquiry: investigating phenology in local campus trees. Science 24, 932-933.

Marbach-Ad G (2004). Expectations and difficulties of first year college students in biology. J Coll Sci Teach 33, 18-23.

Mayer RE (2009). Multimedia Learning, 2nd ed., New York: Cambridge University Press.

Mayer RE, Sims VK (1994). For whom is a picture worth a thousand words? Extensions of dual-coding theory of multimedia learning. J Educ Psychol 86, 389-401.

Patrick P, Tunnicliffe SD (2011). What plants and animals do early childhood and primary students' name? Where do they see them? J Sci Educ Technol 20, 630-642.
Pavio A (1986). Mental Representations: A Dual Coding Approach, Oxford, UK: Oxford University Press.

Potter MC (1976). Short-term conceptual memory for pictures. J Exp Psychol Hum Learn 2, 509-522.

Potter MC, Wyble B, Hagmann CE, McCourt ES (2013). Detecting meaning in RSVP at 13 ms per picture. Atten Percept Psychophys 76, 270-279.

Quinn GP, Keough MJ (2002). Experimental Design and Data Analysis for Biologists, Cambridge, UK: Cambridge University Press.

Raymond JE, Shapiro KL, Arnell KM (1992). Temporary suppression of visual processing in an RSVP task: an attentional blink? J Exp Psychol Hum Percept Perform 18, 849-860.

Raymond JE, Shapiro KL, Arnell KM (1995). Similarity determines the attentional blink. J Exp Psychol Hum Percept Perform 21, 653662.

Schussler EE, Link-Pérez MA, Weber KM, Dollo VH (2010). Exploring plant and animal content in elementary science textbooks. J Biol Educ $44,123-128$.

Schussler EE, Olzak L (2008). It's not easy being green: student recall of plant and animal images. J Biol Educ 42, 112-119.

Shapiro KL, Arnell KM, Raymond JE (1997). The attentional blink. Trends Cogn Sci 1, 291-296.

Sharan L, Rosenholtz R, Adelson EH (2009). Material perception: what can you see in a brief glance? J Vision 9, 784

Uno G (1994). The state of precollege botanical education. Am Biol Teach 56, 263-267.

Uno G (2009). Botanical literacy: what and how should students learn about plants? Am J Bot 96, 1753-1759.

Vul E, Nieuwenstein M, Kanwisher N (2008). Temporal selection is suppressed, delayed, and diffused during the attentional blink. Psychol Sci 19, 55-61.

Wandersee JH (1986). Plant or animals-which do junior high school students prefer to study? J Res Sci Teach 23, 415426.

Wandersee JH, Schussler EE (1999). Preventing plant blindness. Am Biol Teach 61, 84-86.

Wandersee JH, Schussler EE (2001). Towards a theory of plant blindness. Plant Sci Bull 47, 2-9. 